\title{
Surprising shrinkage of expanding gels under an external load
}

\author{
SEON JEONG KIM${ }^{1}$, GEOFFREY M. SPINKS²*, SHONA PROSSER², PHILIP G. WHITTEN², \\ GORDON G. WALLACE ${ }^{2}$ AND SUN I. KIM ${ }^{1}$ \\ ${ }^{1}$ Department of Biomedical Engineering, Hanyang University, Seoul 133-791, South Korea \\ ${ }^{2}$ ARC Centre of Excellence in Electromaterials Science and Intelligent Polymer Research Institute, University of Wollongong, Northfields Ave, Wollongong, NSW 2522, Australia \\ *e-mail: gspinks@uow.edu.au
}

H ydrogels are fascinating and useful in that they can show large volume changes in response to various stimuli, such as temperature or chemical environment ${ }^{1}$. Here we report the peculiar observation that chemically crosslinked hydrogels that normally expand owing to a change in electrolyte $\mathrm{pH}$ can be made to shrink in certain circumstances. Specifically, these hydrogels contract when tested at a constant compressive force and subjected to a $\mathrm{pH}$ change that causes expansion in the absence of the applied load. When tested under tension, the gels always expand. Although the effects of external stress on the swelling of gels is known ${ }^{2-4}$, the concomitant change in gel mechanical properties during $\mathrm{pH}$ switching was found to be a more dominant effect in our studies. However, existing mechanical models ${ }^{5,6}$ used to predict dimensional changes in actuator materials could not explain both the tensile and compression results. In addition, we show that the friction between metal plates of the apparatus and the gel is a key factor in explaining the contractile actuation under compressive loads. The observations reported in this paper are important for the successful design and use of hydrogel actuators in devices such as valves for microfluidics.

Volume changes occur in polyelectrolyte hydrogels when the thermodynamic balance between expansion forces (electrostatic repulsion and osmotic pressure of counterions) and contractile forces (stretching of elastically active network chains) is shifted by changes in the chemical environment. Hydrogel 'artificial muscles $^{37}$ can generate huge dimensional changes in response to changes in temperature ${ }^{8}, \mathrm{pH}^{9}$, solvent ${ }^{10}$ and electric fields ${ }^{11}$. Polymer conformation changes are also accompanied by large changes in equilibrium water content (EWC) of the gel, which can conveniently be measured gravimetrically. Many examples of changes in EWC with changes in $\mathrm{pH}$ are available in the literature, for example in the gels based on chitosan ${ }^{12}$.

Various applications for actuating gels have been proposed, with the microscopic devices seeming most promising owing to their reasonable response times ${ }^{13}$. In practical applications, the influence of the external stresses on the operation of actuators cannot be neglected. Changes in mechanical properties such as
Young's modulus has been shown to significantly affect actuation strains ${ }^{5}$. Externally applied stress has also been shown to change the EWC of gels ${ }^{2-4}$ and to shift the transition temperature of thermally induced discontinuous phase transitions ${ }^{14-17}$, such as the classical gel collapse that occurs in poly $(N$-isopropylacrylamide $)$ at $33^{\circ} \mathrm{C}$. Practical actuator devices will always be subjected to external loads, so it is important to characterize their response under load.

Our initial tests involved measuring the change in the size of a gel that occurred when the $\mathrm{pH}$ of the surrounding electrolyte was shifted from 7 to 2 using the experimental setup shown in Fig. 1. The gel used was an interpenetrating network of chitosan and poly(vinyl alcohol) $)^{12}$, PVA. The gels were chemically crosslinked by ultraviolet radiation. These gels have been shown to swell at low-pH conditions owing to the protonation of amine groups and as shown by huge increases in $\mathrm{EWC}^{12}$. The inset in Fig. 1 shows photographs of the gel after immersion for $24 \mathrm{~h}$ at $\mathrm{pH} 7$ and $\mathrm{pH} 2$ where the volume change of the unconstrained gel is visually evident.

Figure 1 also shows the actuator test results where the gel dimensions were determined in a series of experiments at different applied external loads. In each case the gel was allowed to equilibrate at $\mathrm{pH} 7$ for $24 \mathrm{~h}$ with no load applied. Next, the desired force was applied followed by changing the electrolyte $\mathrm{pH}$ from 7 to 2 while maintaining the force at a constant level. A mechanical displacement and force sensor/controller system was used in feedback mode to maintain a constant force and measure the changes in sample dimension. In these measurements the deformation caused by the application of the applied load was not considered and only the change in dimension due to the $\mathrm{pH}$ shift is reported in Fig. 1. These dimension changes were quite slow, although it has been shown that much faster actuation times can be achieved in microgels ${ }^{13}$. The effects of load on gel actuation reported here would also apply in microgel systems.

The results given in Fig. 1 illustrate the marked effect that applied load has on the $\mathrm{pH}$-induced actuation. In tension, the axial length of the gel increased at all applied loads when the $\mathrm{pH}$ was switched from 7 to 2 . However, the magnitude of the length strain decreased as the applied tensile stress was increased. In contrast, when a compressive stress was applied the sample 
a
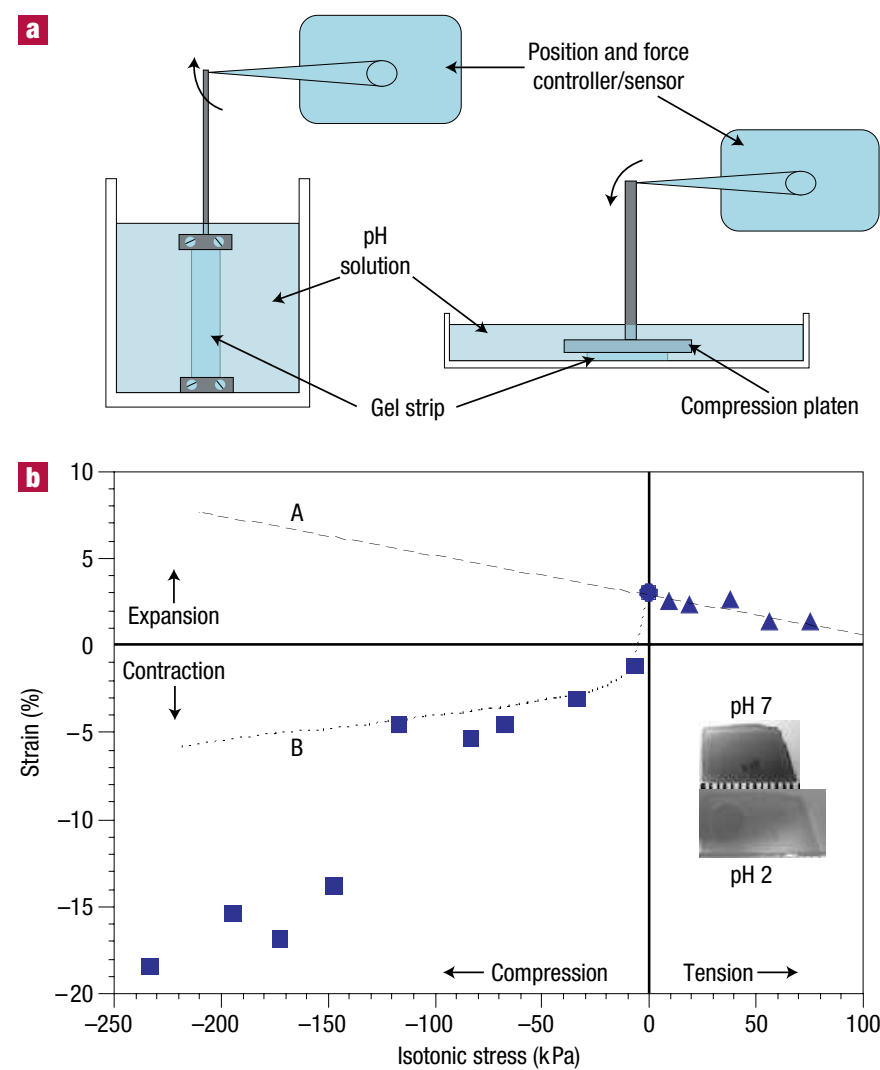

Figure 1 Dimensional changes in chitosan/PVA gel caused by changing the pH of the surrounding solution under isotonic (tension or compression) conditions. a, Testing apparatus. b, Actuation strain measured during $30 \mathrm{~min} \mathrm{pH}$ switching from $\mathrm{pH} 7$ to $\mathrm{pH} 2$ at different isotonic stresses (tension or compression). Compressive stresses and contraction are taken as negative values. The strain is taken from the change in thickness for compression tests (square symbols) and from the change in length for tensile tests (triangle symbols). The circle represents the estimated length change at zero applied stress. The inset shows photographs of a sample equilibrated for $24 \mathrm{~h}$ in each $\mathrm{pH}$ solution (with scale markers of $1 \mathrm{~mm}$ ). Dashed lines $A$ and $B$ are explained in the text.

thickness decreased when the $\mathrm{pH}$ switched from 7 to 2 at a constant compressive load. The $\mathrm{pH}$ induced contraction strain became larger when a higher compressive stress was applied.

Although the tensile actuation results do not follow classical network elasticity behaviour, they can be neatly explained by the concomitant change in modulus that occurs during $\mathrm{pH}$ switching. It has long been known that the equilibrium solvent content of crosslinked rubbers depends on the external stress applied ${ }^{2}$, with a tensile stress causing an increase in solvent content and compressive stresses reducing the solvent content. It has been shown ${ }^{3,4}$ that the effect is exaggerated in charged gels as the applied stress either augments (in tension) or partially cancels (in compression) the swelling due to the osmotic pressure of counterions. Therefore, when a gel is switched from neutral to charged ( $\mathrm{pH} 7$ to $\mathrm{pH} 2$ in our case) the swelling should be greater when a tensile stress is applied compared with the case of zero stress.

Clearly the results shown in Fig. 1 demonstrate the opposite behaviour and highlight that the change in the modulus of the gel during $\mathrm{pH}$ switching dominates the actuation response. As the gels were chemically crosslinked, they behave much like elastic rubbers so that time-dependent (thixotropic) responses as may occur in
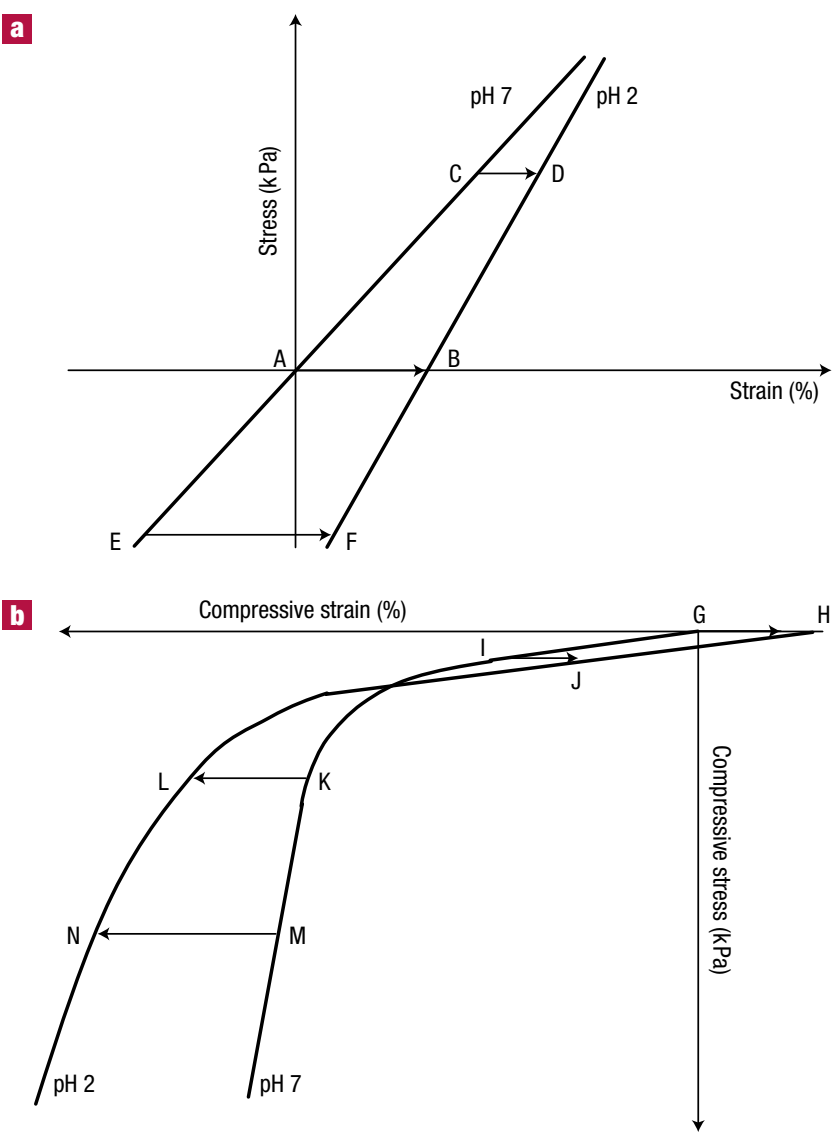

Figure 2 Schematic stress-strain diagrams showing effect of $\mathrm{pH}$ switching on actuation strain at constant tensile and compressive stresses. a, Linear-elastic mechanical response in both tension and compression; $\mathbf{b}$, nonlinear mechanical response typical of thin compliant layers in compression.

physically crosslinked gels can be ignored. Tensile testing of the gels showed that the Young's modulus of the gel increased from 1.0 to $1.2 \mathrm{MPa}$ when the $\mathrm{pH}$ was switched from 7 to 2 . This increase in modulus causes an axial contraction of the gel by an amount proportional to the applied stress ${ }^{6}$. The modulus-induced contraction partially counteracts the natural expansion of the gel leading to lower net expansions when higher tensile stresses were applied. The effect of changing modulus on the $\mathrm{pH}$-induced actuation can be calculated using models described elsewhere ${ }^{5,6}$ and is illustrated schematically in Fig. 2a. The free stroke (at zero stress) is shown by the isotonic tie line between points A and B. The length of the tie line decreases at higher tensile stresses (for example, $\mathrm{C}-\mathrm{D}$ ) as a consequence of the increase in modulus (slope) when the gel is acidified. The dashed line labelled ' $\mathrm{A}$ ' in Fig. 1 is the calculated results, which fit the measured results very well for applied tensile stresses.

Given that the modulus shift dominates the $\mathrm{pH}$ actuation of our gels, the most surprising result was found by comparing the expected behaviour in compression with the observed behaviour. The extrapolation of line A in Fig. 1 to the compressive region shows that a larger and increasing expansion was expected when larger compressive loads were applied to the gel. As described above, however, the opposite behaviour was observed: the gels contracted when the $\mathrm{pH}$ was switched from 7 to 2 at all compressive stress conditions. As shown in Fig. 2a, under ideal circumstances the linear stress-strain curves extend to the compressive region, so 


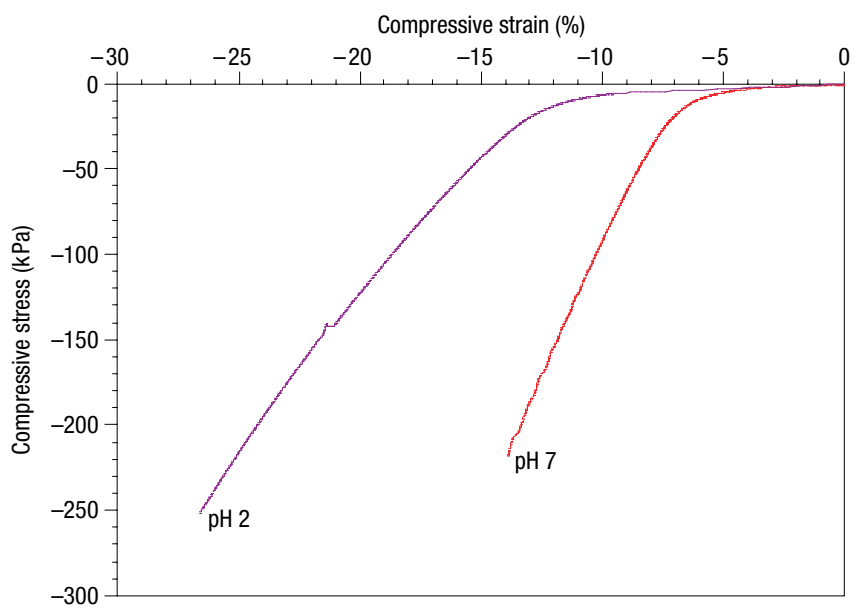

Figure 3 Compressive stress-strain curves for the gels when immersed in different pH solutions. At least three repeat tests were performed with good reproducibility.

that larger expansions are expected when a constant compressive stress is applied (tie-line E-F).

The compression of soft gels can be far from ideal, however, and provides an explanation for the unexpected actuation results in compression. As shown in Fig. 3, the stress-strain curves in compression are highly nonlinear. The form of the compression test results is similar to those in the literature ${ }^{18,19}$, showing a transition from low modulus (slope) to higher modulus with an increase in strain. Of most relevance to the actuation results is that the 'settling strain'19 marking the transition from low to high modulus was reproducibly larger at $\mathrm{pH} 2$ than at $\mathrm{pH} 7$. As a consequence, the isotonic actuation in compression is now predicted by the tie-lines shown in Fig. 2b. The low-slope region is expanded for clarity. At zero stress the gel expands by $3 \%$ so the $\mathrm{pH} 2$ curve is offset by $+3 \%$ on the strain axis (tie-line $\mathrm{G}-\mathrm{H}$ ). Switching the $\mathrm{pH}$ at very small compressive stresses will result in an expansion as shown by tie-line I-J. However, at moderate compressive stresses the behaviour switches to shrinkage as shown by tie-line $\mathrm{K}-\mathrm{L}$. The amount of shrinkage then increases at higher compressive stresses, as illustrated by tie-line $\mathrm{M}-\mathrm{N}$.

The stress-strain curves of Fig. 3 were used to predict the measured actuation strains given in Fig. 1. The predicted strains at various compressive stresses were determined by using the tie-line method and applying a $+3 \%$ strain adjustment to the $\mathrm{pH} 2$ curve to account for the free expansion. The results are shown in Fig. 1 as dashed line B. A reasonable estimation of the actual measured values was obtained by this simple subtraction method at least until a compressive stress of $-125 \mathrm{kPa}$. Discrepancies at higher compressive stresses may be due to small differences in sample geometry affecting the transition point from low to high modulus. Alternatively, it should be noted that a compressive stress will lead to a reduction in the gel solvent content under equilibrium conditions ${ }^{4}$. Such processes may produce larger than expected gel shrinkage at higher compressive stresses. Importantly, the predicted results now anticipate the unexpected shrinkage of the gels that was observed under compressive loads.

The key factor in explaining the surprising shrinkage during compression actuation testing is the shift in the low-to-high modulus transition point when the $\mathrm{pH}$ was changed. The transition to an apparently higher modulus is well known in compression testing of thin compliant materials and is related to the friction between the platens and the sample preventing the lateral (Poisson's) expansion. The form of the compressive stress-strain curve has been shown to depend on the sample aspect ratio $^{20}$, surface roughness ${ }^{21}$, sample-platen friction and the nature of lubricating films ${ }^{19,21}$. The sharp transition from low to high modulus has been previously investigated and found to shift to higher strains when the platen-sample friction was reduced (for example, by using frictionless air bearings). It is known that the friction of charged gels is lower than their uncharged states ${ }^{22}$, which may explain why the stiffness transition occurs at higher strains in the acidified gels than in the neutral gels. In a separate experiment performed on a stack of five gel sheets at a compressive stress of $-18 \mathrm{kPa}$ the gel was found to expand by $+1.1 \%$ when the $\mathrm{pH}$ was switched from 7 to 2 . The gel stack has a higher aspect ratio, which shifts the modulus transition to higher strains. The observed response can then be simulated by tie-line I-J in Fig. $1 \mathrm{~b}$.

A particularly useful feature of these gels when used as actuators is the increasing strain that is produced at higher applied compressive loads. The higher strains at higher loads also produce significantly higher work per cycle, so that the more the gels are compressed the more effectively and efficiently they operate as contractile actuators. This feature is in contrast to the case in tension and other polymer actuators (notably conducting polymers) where the change in modulus tends to partly cancel the actuation strain so that the net actuation becomes smaller at higher loads ${ }^{6}$. In practical devices, a mechanical system could be devised to ensure the gel always operated under compressive loads and to maximize the actuation strain generated.

\section{METHODS}

The gel was prepared as described elsewhere ${ }^{12}$ by first dissolving PVA (Aldrich) in deionized water (10 wt\%). Acryloyl chloride (Aldrich, $3 \mathrm{wt} \%$ ) and 2,2-dimethoxy-2-phenylacetophenone (Aldrich $1 \mathrm{wt} \%$ ) in tetrahydrofuran were mixed with the PVA solution along with chitosan solution (Jakwang, $3 \mathrm{wt} \%$ in $4 \mathrm{wt} \%$ acetic acid). The ratio of PVA to chitosan was 1:1. The mixed solution was exposed to a $450 \mathrm{~W}$ ultraviolet lamp (Ace Glass, USA) to form the crosslinked gel. The water-swollen gels were approximately $0.65 \mathrm{~mm}$ thick and were cut into squares (approximately $5 \mathrm{~mm} \times 5 \mathrm{~mm}$ ) for compression testing and strips $(15 \mathrm{~mm} \times 8 \mathrm{~mm})$ for tensile testing. The mechanical tests were conducted using either a Dual Mode Lever Arm System (Model 300B, Aurora Scientific) or a Dynamic Mechanical Analyser (TA Instruments). Compressive and tensile strains were calculated from the change in thickness and length divided by the initial thickness/length (after $24 \mathrm{~h}$ equilibration at $\mathrm{pH} 7$ ).

Received 8 December 2004; accepted 17 November 2005; published 25 December 2005 .

References

1. Ilmain, F., Tanaka, T. \& Kokufuta, E. Volume transition in a gel driven by hydrogen-bonding. Nature 349, 400-401 (1991)

2. Treloar, L. R. G. The swelling of cross-linked amorphous polymers under strain. Faraday Trans. 46, 783-789 (1950)

3. Budtova, T. \& Suleimenov, I. Swelling behaviour of a polyelectrolyte network under load. Polymer 38, 5947-5952 (1997)

4. Vervoort, S., Patlazhan, S., Weyts, J. \& Budtova, T. Solvent release from highly swollen gels under compression. Polymer 46, 121-127 (2005).

5. Spinks, G. M. \& Truong, V.-T. Work-per-cycle analysis of electromechanical actuators. Sens. Actuat. A $119,455-461$ (2005)

6. Spinks, G. M., Liu, L., Zhou, D. \& Wallace, G. G. Strain response from polypyrrole actuators under load. Adv. Funct. Mater. 12, 437-440 (2002).

7. Osada, Y., Okuzaki, H. \& Hori, H. A polymer gel with electrically driven motility. Nature 355, 242-244 (1992).

8. Yoshida, R. et al. Comb-type grafted hydrogels with rapid de-swelling response to temperature-changes. Nature 374, 240-242 (1995).

9. Brannon-Peppas, L. \& Peppas, N. A. Equilibrium swelling behaviour of $\mathrm{pH}$-sensitive hydrogels. Chem. Eng. Sci. 46, 715-722 (1991).

10. English, A. E., Tanaka, T. \& Edelman, E. R. Equilibrium and non-equilibrium phase transitions in copolymer polyelectrolyte hydrogels. J. Chem. Phys. 107, 1645-1654 (1997).

11. Osada, Y. \& Hasebe, M. Electrically activated mechanochemical devices using polyelectrolyte gels. Chem. Lett. 9, 1285-1288 (1985).

12. Kim, S. J., Sang, J. P. \& Kim, S. I. Swelling behavior of interpentrating polymer network hydrogel composed of poly(vinyl alcohol) and chitosan. Reactive Funct. Polym. 55, 53-59 (2003).

13. Beebe, D. J. et al. Functional hydrogel structures for autonomous flow control inside microfluidic channels. Nature 404, 588-590 (2000).

14. Suzuki, A. \& Ishii, T. Phase coexistence of neutral polymer gels under mechanical constraint. J. Chem. Phys. 110, 2289-2296 (1999). 
15. Hirotsu, S. \& Onuki, A. Volume phase transition of gels under uniaxial tension. J. Phys. Soc. Jpn $\mathbf{5 8}$ 1508-1511 (1989)

16. Kato, E. Pressure-induced volume phase transition of polyacrylamide gels in acetone-water mixtures. J. Chem. Phys. 113, 1310-1314 (2000)

17. Nasimova, I., Karino, T., Okabe, S., Nagao, M. \& Shibayama, M. Effect of ionization on the temperature- and pressure-induced phase transitions of poly(N-isopropylacrylamide) gels. J. Chem Phys. 121, 9708-9715 (2004)

18. Stammen, J. A., Williams, S., Ku, D. N. \& Gulberg, R. E. Mechanical properties of a novel PVA hydrogel in shear and unconfined compression. Biomaterials 22, 799-806 (2001).

19. El-Aguizy, T., Plante, J.-S., Slocum, A. H. \& Vogan, J. D. Frictionless compression testing usin load-applying platens made from porous graphite aerostatic bearings. Rev. Sci. Instrum. 76, 075108 (2005)

20. Gent, A. N. \& Lindley, P. B. The compression of bonded rubber blocks. Proc. Inst. Mech. Eng. 173, 111-117 (1959)
21. Whiting, C. J., Voice, A. M., Olmsted, P. D. \& McLeish, T. C. B. Shear modulus of polyelectrolyte gels under electric field. J. Phys. Condens. Matter 13, 1381-1393 (2001).

22. Gong, J. P. \& Osada, Y. Surface friction of polymer gels. Prog. Polym. Sci. 27, 3-38 (2001).

\section{Acknowledgements}

The authors wish to thank the Australian Research Council for financial support through the ARC

Centre of Excellence in Electromaterials Science and the Linkage International project funding.

Correspondence and requests for materials should be addressed to G.M.S.

Competing financial interests

The authors declare that they have no competing financial interests.

Reprints and permission information is available online at http://npg.nature.com/reprintsandpermissions/ 